\title{
Protocolo de actuación ante la rosácea en la farmacia comunitaria
}

\section{Antucho Espinosa Suances', Javier Labandeira García²}

1. Licenciado en Farmacia. Farmacéutico Comunitario en A Valenzá, Ourense. Máster en Atención Farmacéutica.

2. Doctor en Medicina. Dermatólogo Adjunto, Servicio de Dermatología, Complejo Hospitalario Universitario de Santiago de Compostela.

Profesor Asociado, Departamento de Dermatología, Facultad de Medicina, Santiago de Compostela.

\section{PALABRAS CLAVE}

Rosácea, dermatosis facial, farmacia comunitaria, farmacéutico comunitario

\section{ABREVIATURAS}

FC: farmacia comunitaria

AF: actuación farmacéutica NRS: National Rosacea

Society

sp: especie

\section{KEYWORDS}

Rosacea, facial dermatoses, community pharmacy, community pharmacist

\section{RESUMEN}

La rosácea es una dermatosis facial inflamatoria, recidivante y crónica, que con frecuencia demanda consulta en la farmacia comunitaria. Orientado hacia la práctica clínica del farmacéutico comunitario, este artículo revisa y sintetiza los conceptos clásicos y los avances más recientes en la comprensión y el tratamiento de esta enfermedad cutánea. Finalmente, propone un protocolo para la asistencia de pacientes con rosácea en la farmacia comunitaria.

\section{Course of action for rosacea in community pharmacy}

\section{ABSTRACT}

Rosacea is a chronic relapsing inflammatory facial dermatosis that demands often attention in the Community Pharmacy. Keeping in mind what could be useful in the clinical practice for the community pharmaceutist, this paper reviews and synthesizes clasical concepts and the most recent advances in the understanding and management of this skin disease. Finally, a protocol for the assistence of patients with rosacea in the Community Pharmacy is proposed.
Recibido: 20/2/2016

Aceptado: $12 / 5 / 2016$

Disponible online: 1-6-2016
Financiación: Ninguna ajena a los autores.

Conflicto de intereses: Los autores declaran no existir conflicto de intereses en relación con el contenido del presente artículo.

Declaración de autoría: Ambos autores participaron por igual en el diseño, la revisión, la redacción del manuscrito y la revisión del contenido final.

Cite este artículo como: Espinosa A, Labandeira J. Protocolo de actuación ante la rosácea en la farmacia comunitaria. Farmacéuticos Comunitarios. 2016 Jun 30; 8(2):28-34. doi:10.5672/FC.21739218.(2016/Nol8).002.05

Autor para correspondencia: Antucho Espinosa Suances (antuchoespinosa@redfarma.org) ISSN 1885-8619 @SEFAC (Sociedad Española de Farmacia Familiar y Comunitaria). Todos los derechos reservados. 


\section{Introducción}

La rosácea es una dermatosis facial que con frecuencia genera numerosas consultas en la farmacia comunitaria (FC) debido a sus síntomas cutáneos y oculares, a los brotes súbitos de lesiones inflamatorias y a la mala tolerancia a cosméticos y otros productos tópicos. Esta demanda ha sido cuantificada recientemente, observándose que la rosácea representa el $4 \%$ de todas las consultas por problemas dermatológicos realizadas en la FC [1].

El término "rosácea” procede del latín rosacēus, que significa "de color parecido al de la rosa” [2], y designa a una enfermedad cutánea frecuente, benigna, que afecta principalmente a personas de edad media y piel clara. Su causa es inflamatoria, pero el mecanismo por el que se desencadena es complejo y no bien conocido, involucrando principalmente alteraciones en la inmunidad innata y en la barrera epidérmica.

Clínicamente, debe considerarse a la rosácea como un síndrome pues puede combinar diversos tipos de alteraciones cutáneas, siendo las más características el eritema persistente con telangiectasias, las crisis vasomotoras y los brotes inflamatorios de pápulas y pústulas. Según el tipo de alteración cutánea que presenta se clasifica en subtipos y estadios.

Habitualmente, su diagnóstico es clínico y evoluciona de forma crónica con brotes y recidivas muy frecuentes. Su tratamiento incluye normas generales, comunes para todos los pacientes, y una terapia, tópica o/y sistémica, específica para cada estadio.

Los siguientes apartados intentan sintetizar de forma sencilla los conocimientos más recientes sobre esta enfermedad dermatológica que puedan ser de utilidad al farmacéutico comunitario. Finalmente, se propone un protocolo o procedimiento de actuación en FC ante una consulta por rosácea.

\section{Epidemiología}

La rosácea es una enfermedad frecuente, con mayor prevalencia en personas de piel clara y una incidencia estimada de $1,65 \times 1.000$ personas-año. En el $80 \%$ de los casos se manifiesta después de los 30 años de edad (con predominio en mujeres) $\mathrm{y}$, sobre todo, a partir de la quinta década (con similar distribución por sexo) [3].

La historia familiar de rosácea y la excesiva exposición solar aumentan el riesgo de padecerla, mientras que el consumo de alcohol, al contrario de lo que tradicionalmente se piensa, sólo la incrementa marginalmente [3].

\section{Etiopatogenia}

El mecanismo exacto por el que se desencadena la rosácea es aún desconocido. Su etiopatogenia es muy compleja y ha sido ampliamente estudiada, considerándose actualmente que esta dermatosis es una enfermedad inflamatoria desencadenada principalmente por una respuesta anormal de la inmunidad innata a diversos estímulos externos, asociada a otras alteraciones neurovasculares y de la barrera epidérmica.

Los siguientes apartados resumen los hallazgos más recientes sobre los mecanismos implicados en el desarrollo de la enfermedad.

\section{Alteraciones neurovasculares}

Consideradas durante años como la principal causa de la rosácea, actualmente se postula que sólo estarían implicadas en su estadio inicial, durante la activación de la cascada inflamatoria que posteriormente induce las lesiones clínicas y la progresión de la enfermedad [4].

\section{Alimentos}

En el pasado se ha dado mucha importancia a los factores alimentarios; actualmente, no se considera que tengan relación con el desarrollo de la enfermedad. La única norma que persiste es la de evitar todo lo que cause vasodilatación facial (alimentos o bebidas muy calientes, especias, alcohol) [3].

\section{Alteraciones genéticas}

En la rosácea existe predisposición familiar y racial, siendo más frecuente en personas de piel clara (fototipos I y II de Fitzpatrick de origen celta y del norte de Europa) que en asiáticos y sujetos de piel oscura. Sin embargo, la base genética de la enfermedad no es bien conocida $[3,4]$.
4. Inmunidad innata, péptidos proinflamatorios y alteración de la barrera epidérmica

Estudios moleculares recientes han mostrado que la calicreína $5 \mathrm{y}$ la catelicidina LL-37, péptidos que modulan la respuesta inmune innata ante factores medioambientales, presentan mayor actividad en piel con rosácea que en piel normal, e inician una respuesta inflamatoria exagerada ante diversos estímulos [5,6]. Por otra parte, esta expresión exagerada de la molécula proinflamatoria catelicidina y la alteración de la barrera cutánea sólo se observa en la cara en pacientes con rosácea, contrariamente a enfermedades como la dermatitis atópica en la que se ve afectada toda la piel.

\section{Infecciones}

Los principales agentes tradicionalmente implicados en la etiopatogenia de la rosácea son demodex, estafilococo epidermidis y helicobacter pylori. Aunque su relación con la enfermedad sigue a debate, no existen actualmente suficientes evidencias para aceptar una etiología microbiana.

La posible relación del ácaro humano Demodex sp. (parásito obligatorio del folículo pilosebáceo humano) con la rosácea ha sido estudiada extensamente pero sigue siendo cuestionada [7]. Por otra parte, las infecciones por Demodex (demodecidosis) pueden originar diversas dermatosis inflamatorias que simulan foliculitis bacterianas o rosácea, dificultando aún más la valoración de su papel etiopatogénico en la rosácea genuina. El estafilococo epidermidis podría estar implicado en los brotes de lesiones inflamatorias. Finalmente, la posible asociación de Helicobacter pylori y rosácea, tema de estudio y debate en las últimas dos décadas, sigue siendo controvertida [3].

\section{Enfermedades asociadas}

Se ha postulado una posible relación de la rosácea con enfermedades psiquiátricas y alteraciones psicológicas; sin embargo, estudios recientes no han observado relación entre la depresión (u otros desórdenes afectivos) $\mathrm{y}$ la incidencia de rosácea [8]. Sí ha sido observado un mayor riesgo de padecerla en pacientes con enfermedades cardiovasculares, migraña, rinosinusitis 
crónica e hipervitaminosis D, y un menor riesgo en pacientes con diabetes mellitus de larga evolución [9].

\section{Manifestaciones clínicas}

La rosácea es un síndrome de evolución crónica, caracterizado por accesos vasomotores, lesiones cutáneas y lesiones oculares. Como en toda enfermedad dermatológica, para describir su clínica es necesario mencionar las lesiones elementales primarias y secundarias que la definen. En la rosácea son: eritema (transitorio y permanente), telangiectasias, pápulas, pústulas, edema y esclerosis. Las lesiones se distribuyen de forma simétrica y afectan típicamente a las áreas convexas centrofaciales (frente, glabela, nariz, áreas malares $\mathrm{y}$, con menor frecuencia, el mentón) [10].

\section{Síntomas cutáneos}

En la mayoría de los casos de rosácea, el paciente se autodefine como "de piel sensible", con sensación de ardor, quemazón, pinchazos o picazón en la cara y también padece intolerancia a cosméticos habituales, lo que suele agravar dicha sintomatología.

\section{Lesiones cutáneas primarias}

Constituyen las principales manifestaciones clínicas de la enfermedad. La presencia de una o más de ellas con distribución centrofacial es muy sugestiva de rosácea.

\section{Eritema transitorio (rubor)}

Denominado en la literatura anglosajona flush/flushing. El paciente refiere una historia de episodios frecuentes de eritema facial desencadenado por estímulos como el calor (al cocinar, en ambientes cerrados) o el alcohol. Es un dato clínico muy útil para el diagnóstico, pero debe recordarse que en la rosácea, a diferencia de lo que ocurre en personas sanas, en las que el rubor desaparece en unos minutos, persiste más tiempo, pudiendo durar horas o días.

\section{Eritema permanente centrofacial \\ Es el signo clínico más frecuente de esta enfermedad (figura 1).}

\section{Telangiectasias centrofaciales}

También frecuentes, pero pueden existir en otras dermatosis por lo que deben valorarse en el contexto de los demás sintomas y signos clínicos de la rosácea (figura 1).

\section{Pápulas y pústulas}

Son dos tipos de lesiones cutáneas que surgen en el transcurso de la enfermedad. "Pápula" es una lesión elemental primaria de contenido sólido ("maciza", sin contenido líquido ni “cabeza” de pus), palpable, hemisférica, $<1 \mathrm{~cm}$, que cura sin cicatriz; de color rojo en la rosácea (figura 2). "Pústula" es una lesión elemental primaria de contenido purulento, de 1 a $3 \mathrm{~mm}$,

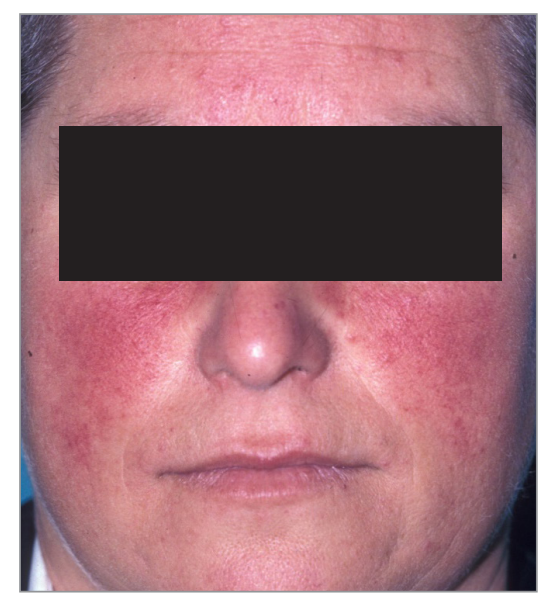

Figura 1 Eritema permanente $y$ telangiectasias centrofaciales, respetando las áreas periorificiales (ojos, boca) y el mentón (subtipo 1 de rosácea)

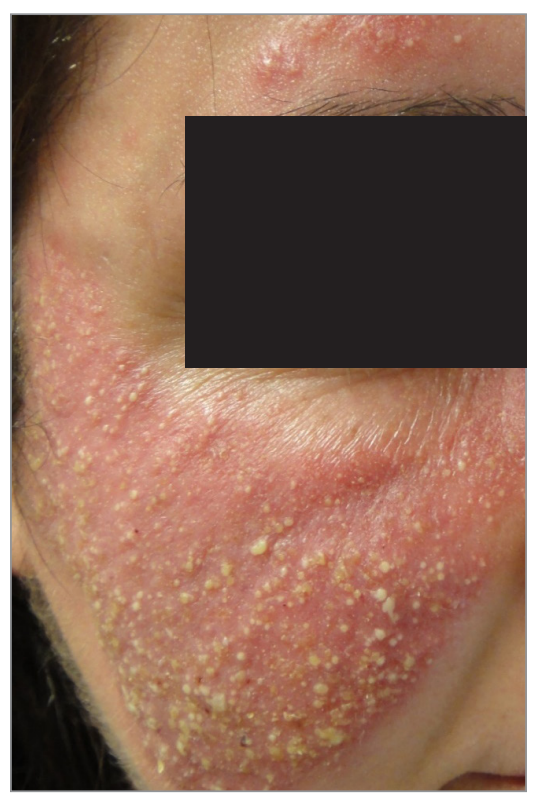

Figura 3 Múltiples pústulas blancoamarillentas sobre una base eritematosa centrofacial que respeta las áreas periorificiales y el mentón (subtipo 2 de rosácea) con un pequeño halo eritematoso y color blanco-amarillento (figura 3).

El paciente sufre brotes de pápulas y pústulas, escasas o múltiples, distribuidas de forma simétrica en una o varias zonas del área centrofacial, respetando típicamente las áreas periorificiales (ocular, bucal) y con frecuencia el mentón (figuras 2 y 4). Los brotes son típicos y duran semanas o meses. Normalmente muestran ambos tipos de lesiones pero el paciente también puede mostrar sólo múltiples pápulas o solo pústulas.

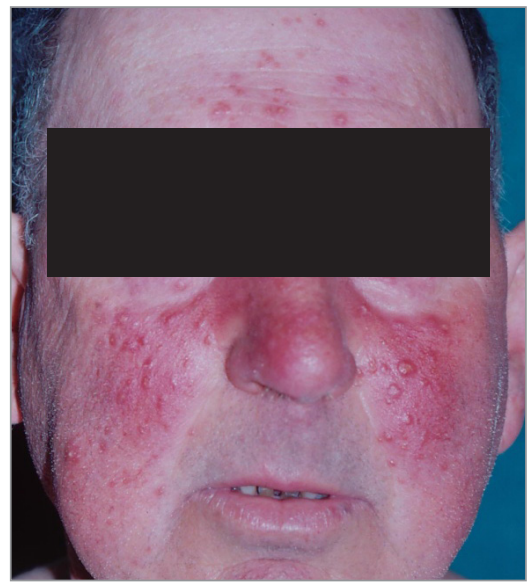

Figura 2 Pápulas eritematosas sobre áreas de eritema permanente y telangiectasias centrofaciales, respetando las regiones periorificiales y el mentón (subtipo 2 de rosácea)

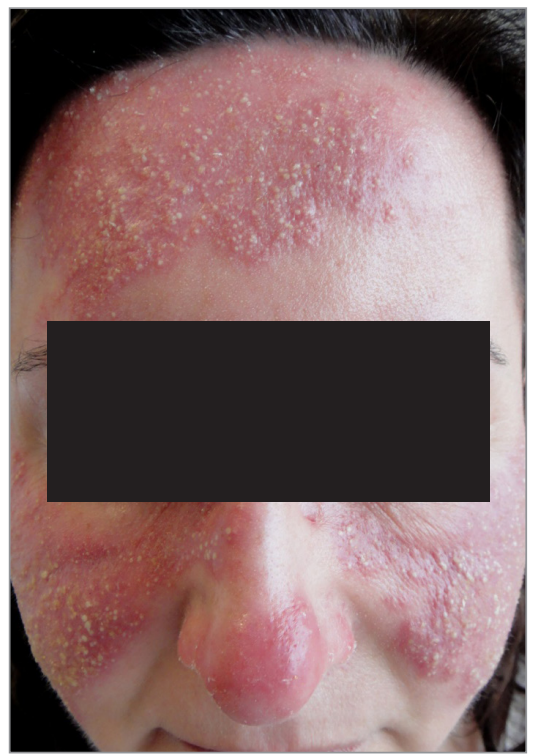

Figura 4 Imagen típica de rosácea, con múltiples pápulas y pústulas sobre áreas de eritema distribuidas de forma centrofacial y simétrica, respetando zona periorificial y mentón (subtipo 2 de rosácea) 


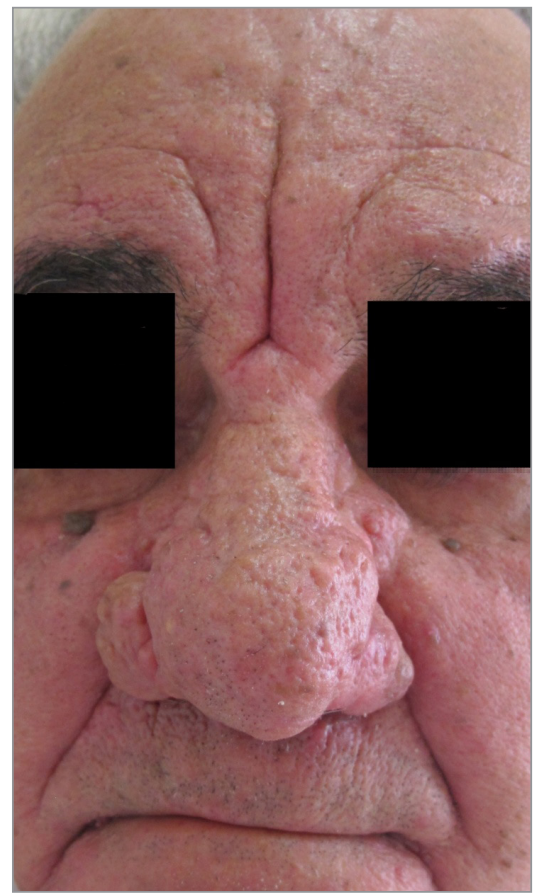

Figura 5 Engrosamiento de la piel nasal (rinofima, subtipo 3 de rosácea)

\section{Lesiones cutáneas secundarias}

Habitualmente no existen aisladas, sino asociadas a alguna de las lesiones primarias descritas. Las principales lesiones secundarias son sequedad cutánea centrofacial, placas (área cutánea engrosada, palpable, $>1 \mathrm{~cm}$, como un sumatorio de pápulas) eritematosas, edema sólido y permanente (sin fóvea) y fimas. Las fimas (del griego phyma, masa o bulbo) son engrosamientos localizados de la piel facial, más frecuentemente en la nariz (rinofima) (figura 5).

\section{Clínica ocular}

Se denomina "rosácea oftálmica". Asociada con frecuencia a las manifestaciones cutáneas de la enfermedad, suele ser bilateral y poco sintomática [11]. La clínica es muy variable, desde sólo sensación de ardor y quemazón a signos de conjuntivitis ("ojo rojo") o afectación corneal (dolor, lagrimeo y fotofobia) (figura 6).

\section{Clasificación clínica de la rosácea}

Debido a la variabilidad de sus manifestaciones existe el riesgo de calificar como rosácea a diversas alteraciones cutáneas faciales con similitudes clínicas. Para evitarlo, diversas escuelas dermatológicas han

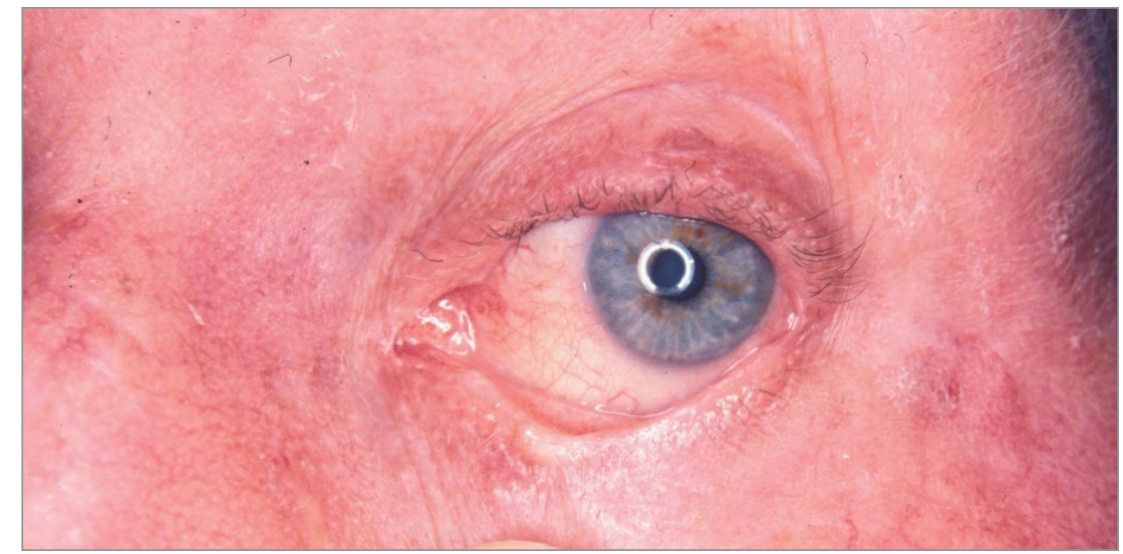

Figura 6 Conjuntivitis en el contexto de una rosácea con 2 subtipos asociados. La clínica ocular la incluye en el subtipo 4 (rosácea oftálmica) y la clínica cutánea en el subtipo 1 (eritema y telangiectasias, sin lesiones inflamatorias)

realizado clasificaciones que facilitan su diagnóstico. Actualmente, la más difundida es la desarrollada en 2002 por la National Rosacea Society (NRS) de Norteamérica [12]. En dicha clasificación, la enfermedad se subdivide en cuatro subtipos, pudiendo iniciarse en cualquiera de ellos o presentar manifestaciones de varios subtipos al mismo tiempo (tabla 1).

A nivel práctico, puede ser de especial interés para el farmacéutico comunitario conocer que, de los cuatro posibles subtipos de rosácea, casi la totalidad de los pacientes que consultan en la farmacia pertenecen al subtipo 1 (57\%) o al 2 (35\%) [1].

\section{Subtipo 1 (Rosácea}

eritematotelangiectásica)

Son pacientes que presentan sólo manifestaciones vasculares, como accesos vasomotores (rubor facial), telangiectasias o eritema centrofacial permanente, asociadas o no a sensación de ardor e intolerancia a cosméticos habituales (figura 1).

Tabla 1 Clasificación de la rosácea por subtipos. Variedades y exclusiones (NRS)

\begin{tabular}{l}
\hline SUBTIPOS \\
S 1 - R Eritematotelangiectásica \\
S 2 - R Pápulo-pustulosa \\
S 3 - R con Fimas \\
S 4 - R Ocular \\
VARIEDADES CLÍNICAS \\
R Granulomatosa \\
EXCLUSIONES \\
R fulminans \\
Dermatitis inducida por esteroides \\
Dermatitis perioral
\end{tabular}

S: subtipo; R: rosácea.

\section{Subtipo 2 (Rosácea}

pápulopustulosa)

Es el subtipo más característico y conocido de la enfermedad. Son pacientes que, además del eritema persistente del subtipo 1, presentan los brotes recurrentes ya descritos de pápulas (figura 2) y/o pústulas (figuras 3 y 4).

\section{Subtipo 3 (Rosácea con fimas)}

Es poco frecuente, prácticamente solo en varones y después de la cuarta década (figura 5).

\section{Subtipo 4 (Rosácea oftálmica)}

Caracterizada por las manifestaciones oculares mencionadas. Habitualmente se asocia a la clínica cutánea pero también puede manifestarse de forma aislada (figura 6).

\section{Consideraciones clínicas}

Los cuatro subtipos descritos, solos o combinados, constituyen la enfermedad conocida como rosácea. Sin embargo, la síntesis clínica previa no estaría completa sin dos importantes consideraciones: las variedades y las exclusiones (procesos clínicamente diferentes a la rosácea pero considerados como variedades de la misma y procesos clínicamente similares pero considerados como entidades diferentes).

\section{Variedades clínicas de la rosácea}

A lo largo de los años han sido descritas diversas dermatosis faciales que, si bien no presentan las manifestaciones clínicas propias de los cuatro subtipos de rosácea, se consideraban como formas clínicas especiales de la misma. Actualmente, la NRS sólo reconoce una variante (poco frecuente): 
la denominada "Rosácea granulomatosa” [12].

\section{Exclusiones}

Son enfermedades clasificadas en el pasado como variedades de rosácea, pero que actualmente se consideran entidades independientes debido a que los datos existentes son insuficientes para englobarlas en el espectro de la rosácea. Estas exclusiones afectan principalmente a las denominadas "Rosácea fulminans”, "Rosácea inducida por esteroides" y "Dermatitis perioral" [11]. Entre ellas destaca la rosácea inducida por corticosteroides, tanto tópicos como nebulizados nasalmente, que se manifiesta por brotes de pápulas y pústulas sobre piel eritematosa como respuesta inflamatoria a un tratamiento prolongado con el fármaco [12].

\section{Dermatopatología}

El diagnóstico de la rosácea es clínico y raramente es necesario realizar una biopsia y estudio histológico para confirmarlo (excepto en la forma granulomatosa o en formas atípicas del subtipo 1 que simulan un lupus eritematoso). Los hallazgos histológicos pueden simular otras dermatosis crónicas y varían según el subtipo clínico de la rosácea [13].

\section{Diagnóstico diferencial}

En la mayoría de los casos, la presentación clínica de la rosácea es característica y suficiente para el diagnóstico, sin originar confusión con otras enfermedades. Teóricamente, pueden presentar cierta similitud clínica con ella y obligar a un diagnóstico diferencial: acné vulgar (el diagnóstico diferencial más importante), dermatitis perioral, erupciones acneiformes inducidas por corticosteroides, lupus eritematoso y, en general, procesos que cursan con accesos vasomotores faciales (premenopausia, síndrome carcinoide, mastocitosis, estenosis mitral y policitemia), con eritema facial permanente (principalmente etilismo crónico) o con telangiectasias faciales (cuperosis común, esclerodermia sistémica [14]. Muy raramente, formas atípicas de rosácea podrían necesitar diagnóstico diferencial con dermatomiositis, sífilis, tuberculosis, sarcoidosis, leucemia cutis o linfomas B [15].

\section{Tratamiento}

La rosácea es un proceso crónico pero los tratamientos actuales suelen ser eficaces para detener los brotes y la evolución natural de la enfermedad. El primer paso para elegir la terapéutica adecuada es definir el principal subtipo de rosácea que afecta al paciente, y luego si existe sintomatología asociada.

El tratamiento consiste en medidas higiénico-sanitarias, fármacos tópicos o/y sistémicos, y terapias físicas. Respecto al tratamiento farmacológico, actualmente sólo han sido aprobados cuatro medicamentos tópicos (metronidazol, ácido azelaico, brimonidina e ivermectina) y uno oral (doxiciclina; recientemente también en su dosis submicrobiana de $40 \mathrm{mg}$ de liberación retardada). Sin embargo, en la práctica clínica se utilizan habitualmente otras medicaciones tópicas y sistémicas, principalmente en casos refractarios, incluso fármacos no comercializados en España, como la ivermectina oral.

\section{Medidas higiénico-sanitarias}

Como norma general, la piel del paciente con rosácea tiene una mala tolerancia a las substancias externas. Por lo tanto, hay que evitar todo producto, cosmético o farmacológico, que potencialmente pueda irritarla: acetona, alfa-hidroxiácidos, propilenglicol, lauril sulfato sódico, liberadores de formaldehído, cinamatos, benzofenonas, mentol, alcohol bencílico o alcanfor. Esto incluye jabones agresivos, sustancias astringentes y vehículos con alcohol o fragancias. Los fotoprotectores (recomendables) se toleran mejor si contienen sólo filtros físicos. Para camuflar el eritema y las telangiectasias pueden utilizarse bases cosméticas de tono verde.

Respecto a las medidas higiénicas, ayuda a disminuir la frecuencia e intensidad de los accesos vasomotores utilizar agua templada para el aseo facial (nunca muy fría o muy caliente), un limpiador suave y líquido, tocar la cara sólo con la punta de los dedos y no con toallitas de limpieza o esponjas, y secarse por absorción (presionando en lugar de frotar) con una toalla de algodón [16].

Finalmente, las medidas climáticas consisten en evitar una exposición excesiva al sol, al frío o al calor.

\section{Fármacos tópicos}

Para tratar los brotes de lesiones inflamatorias pueden utilizarse antibióticos (clindamicina, eritromicina), antimicóticos (imidazoles), ácido azelaico y antiparasitarios (lindane, permetrina e ivermectina) [17].

El eritema facial de la rosácea puede ser tratado con los agonistas tópicos de los receptores $\alpha$-adrenérgicos brimonidina y oximetazolina (no comercializado) [18].

\section{Fármacos sistémicos}

Como se ha mencionado, la única medicación oral aprobada para el tratamiento de la rosácea es la doxiciclina, en dosis de 50-100 mg, o en dosis submicrobianas de $40 \mathrm{mg}$ [19]. Otros fármacos orales alternativos son isotretinoina, azitromicina, metronidazol e ivermectina. Tanto doxiciclina como isotretinoina han mostrado eficacia no sólo para suprimir los brotes de lesiones inflamatorias (pápulas y pústulas) sino también para reducir la angiogénesis (lesiones vasculares) propia de la enfermedad [20].

\section{Terapias físicas}

Las principales terapias físicas utilizadas en la rosácea son la cirugía y el láser. Son seguras y eficaces para tratar las manifestaciones clínicas de los subtipos 1 (rosácea eritematotelanguiectásica) y 3 (rosácea con fimas) $[21,22]$.

\section{Protocolo de actuación farmacéutica en la rosácea}

La figura 7 muestra un algoritmo que, de forma sencilla, pretende facilitar la actuación del farmacéutico. Como casi la totalidad de los pacientes con rosácea, que demandan consulta farmacéutica, pertenecen a los subtipos 1 o 2 [1], dicho algoritmo se limita a ambos subtipos, excluyendo la rosácea con fimas y la rosácea oftálmica, que en todo caso se derivarían al médico.

Ante un paciente con una dermatosis facial de aspecto inflamatorio, el farmacéutico debe en primer lugar, averiguar si existe un diagnóstico previo de rosácea. En caso afirmativo, o si el cuadro clínico es muy sugestivo de la misma, el farmacéutico 


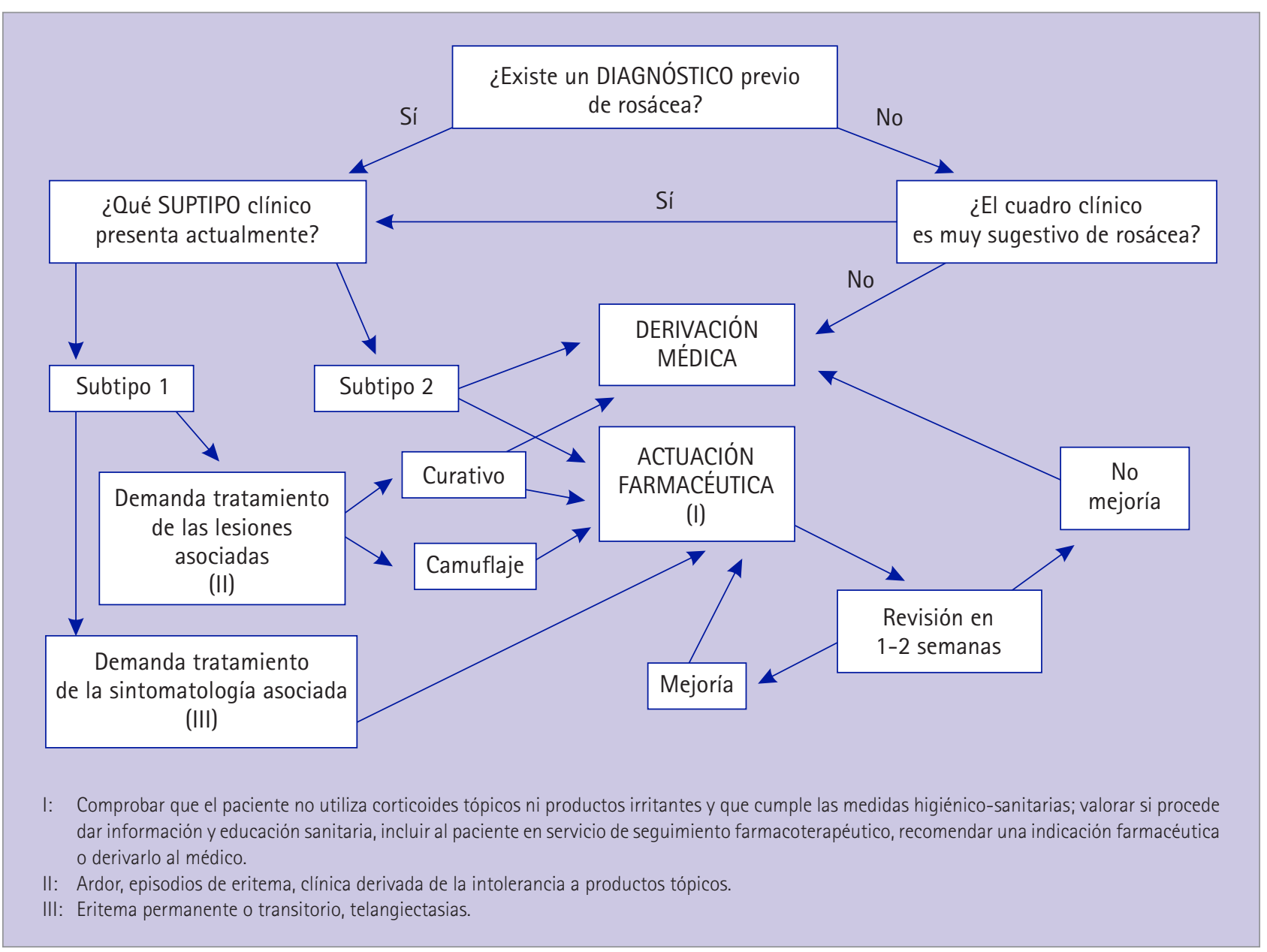

Figura 7 Algoritmo ante una consulta farmacéutica por una dermatosis facial

debe en segundo lugar plantearse qué subtipo clínico presenta el paciente. En caso negativo, se derivará al médico.

Si es un subtipo 1 y el motivo de consulta es la sintomatología asociada (ardor, episodios de eritema, clínica derivada de la intolerancia a productos tópicos), o el deseo de camuflar las lesiones propias de dicho estadio, se procederá a una actuación por parte del farmacéutico.

Dicha actuación consistirá en comprobar que el paciente no utiliza medicamentos tópicos contraindicados en la rosácea (corticosteroides) ni productos potencialmente irritantes y que cumple las medidas higiénico-sanitarias ya mencionadas, prestando especial atención a los productos cosméticos, de higiene y de fotoprotección [16]. Si en la actuación previa se detecta que el paciente no cumple alguno de los supuestos anteriores, el farmacéutico le suministrará la información y educación sanitarias necesarias para corregir dicha situación. También valorará si procede realizar otra actuación, como incluir al pa- ciente en el servicio de seguimiento farmacoterapéutico de la farmacia, recomendar medicamentos o productos sanitarios de indicación farmacéutica o derivarlo al médico [23].

Si el paciente demanda un tratamiento curativo de las lesiones asociadas al subtipo 1 (eritema permanente o transitorio, telangiectasias), o bien presenta un subtipo 2, además de realizar alguna o varias de las actuaciones anteriores, estaría indicada la derivación al médico.

En todo caso, los resultados de la intervención farmacéutica se evaluarán al cabo de 1 o 2 semanas, derivando al médico en caso de no observarse mejoría.

\section{Referencias bibliográficas}

1. González Hernández P, González Rodríguez A, Alcocer Muñoz MJ, González Borrego A, Ibáñez Gandasegui A. Indicación farmacéutica en patologías dermatológicas. Farmacéuticos Comunitarios. 2014 [Internet]; 6(Supl 1). http:// www.farmaceuticoscomunitarios.org/ es/journal-article/indicacion-farmaceutica-patologias-dermatologicas
2. Real Academia Española. Diccionario de la lengua española. 22 edición, 2001 [Internet] [acceso 16 de julio de 2015]. Disponible en: http://lema.rae. es/drae/?val=ros\%C3\%A1cea.

3. Tan J, Berg M. Rosacea: current state of epidemiology. J Am Acad Dermatol. 2013; 69(Suppl 1): S27-35. doi:10.1016/j.jaad.2013.04.043

4. Cribier B. Physiopathology of rosacea. Ann Dermatol Venereol. 2014; 141(Suppl 2):S158-164. doi:10.1016/ S0151-9638(14)70153-X

5. Salzer S, Kresse S, Hirai Y, Koglin S, Reinholz M, Ruzicka T et al. Cathelicidin peptide LL-37 increases UVB-triggered inflammasome activation: possible implications for rosacea. J Dermatol Sci. 2014; 76(3):173-179. doi:10.1016/j.jdermsci.2014.09.002

6. Two AM, Del Rosso JQ. Kallikrein 5-mediated inflammation in rosacea: clinically relevant correlations with acute and chronic manifestations in rosacea and how individual treatments may provide therapeutic benefit. J Clin Aesthet Dermatol. 2014; 7(1):20-25.

7. Chen W, Plewig G. Human demodicosis: revisit and a proposed classification. Br J Dermatol. 2014; 170(6):12191225. doi:10.1111/bjd.12850 
8. Spoendlin J, Bichsel F, Voegel JJ, Jick SS, Meier CR. The association between psychiatric diseases, psychotropic drugs and the risk of incident rosacea. $\mathrm{Br} \mathrm{J}$ Dermatol. 2014; 170:878-883. doi:10.1111/ bjd.12734

9. Spoendlin J, Voegel JJ, Jick SS, Meier CR. Risk of rosacea in patients with diabetes using insulin or oral antidiabetic drugs. J Invest Dermatol. 2013; 133:27902793. doi:10.1038/jid.2013.225

10. Schmutz JL. Signs and symptoms of rosácea. Ann Dermatol Venereol. 2014; 141(Suppl 2):S151-S157. doi:10.1016/S0151-9638(14)70152-8

11. Vieira AC, Mannis MJ. Ocular rosacea: common and commonly missed. J Am Acad Dermatol. 2013; 69(6 Suppl 1):S36-S41. doi:10.1016/j. jaad.2013.04.042

12. Wilkin J, Dahl M, Detmar M, Drake L, Feinstein A, Odom R et al. Standard classification of rosacea: Report of the National Rosacea Society Expert Committee on the Classification and Staging of Rosacea. J Am Acad Dermatol 2002; 46(4):584-587. doi:10.1067/mjd.2002.120625

13. Cribier B. Rosacea under the microscope: characteristic histological findings. J Eur Acad Dermatol Venereol. 2013; 27(11):1336-1343. doi:10.1111/jdv.12121
14. Olazagasti J, Lynch P, Fazel N. The great mimickers of rosacea. Cutis. 2014; 94(1):39-45.

15. Barzilai A, Feuerman H, Quaglino P, David M, Feinmesser M, Halpern M et al. Cutaneous B-cell neoplasms mimicking granulomatous rosácea or rhinophyma. Arch Dermatol. 2012; 148(7):824-831. doi:10.1001/archdermatol.2011.3575

16. Del Rosso JQ, Thiboutot D, Gallo R, Webster G, Tanghetti E, Eichenfield L et al. Consensus recommendations from the American Acne Ctamp; Rosacea Society on the management of rosacea, part 1: a status report on the disease state, general measures, and adjunctive skin care. Cutis. 2013; 92(5):234-240.

17. Del Rosso JQ, Thiboutot D, Gallo R, Webster G, Tanghetti E, Eichenfield $\mathrm{L}$ et al. Consensus recommendations from the American Acne Etamp; Rosacea Society on the management of rosacea, part 2: a status report on topical agents. Cutis. 2013; 92(6):277-284.

18. Johnson AW, Johnson SM. The Role of Topical Brimonidine Tartrate Gel as a Novel Therapeutic Option for Persistent Facial Erythema Associated with Rosacea. Dermatol Ther (Heidelb). 2015 Jun 26. [Epub ahead of print].

19. Del Rosso JQ, Webster GF, Jackson M, Rendon M, Rich P, Torok H et al. Two randomized phase III clinical trials evaluating anti-inflammatory dose doxycycline (40-mg doxycycline, USP capsules) administered once daily for treatment of rosacea. J Am Acad Dermatol 2007; 56(5):791-802. doi:10.1016/j.jaad.2006.11.021

20. Del Rosso JQ1, Thiboutot D, Gallo R, Webster G, Tanghetti E, Eichenfield LF et al. Consensus recommendations from the American Acne Ctamp; Rosacea Society on the management of rosacea, part 3: a status report on systemic therapies. Cutis. 2014; 93(1):1828.

21. Tanghetti E, Del Rosso JQ, Thiboutot D, Gallo R, Webster G, Eichenfield LF, et al. Consensus recommendations from the American acne \&t amp; rosacea society on the management of rosacea, part 4: a status report on physical modalities and devices. $\mathrm{Cu}-$ tis. 2014; 93(2):71-76.

22. Mazer JM. Role of laser in the treatment of rosacea. Ann Dermatol Venereol. 2014; 141(Suppl 2):S175-S178. doi:10.1016/S0151-9638(14)70156-5

23. Foro de Atención Farmacéutica en Farmacia Comunitaria. Guía Práctica para los Servicios de Atención Farmacéutica en Farmacia Comunitaria. Madrid: Consejo General de Colegios Oficiales de Farmacéuticos; 2010. 